\title{
How to Distinguish Non-Metastatic Rhabdoid Tumor from Wilms Tumor in Young Infants Preoperatively: A Case Report and Literature Review
}

\author{
Sahla Sellami $^{{ }^{*}}$, Saloua Ammar ${ }^{1}$, Saif Ben Hammouda ${ }^{2}$, Chiraz Chaari ${ }^{2}$, Mohamed Zouari ${ }^{1}$, Hayet Zitouni ${ }^{1}$, \\ Tahya Sellami $^{2}$, Mahdi Ben Dhaou ${ }^{1}$ and Riadh Mhiri ${ }^{1}$
}

${ }^{1}$ Department of Pediatric Surgery, Hedi Chaker Hospital, University of Sfax, Tunisia

${ }^{2}$ Department of Histopathology Habib Bourguiba Hospital, University of Sfax, Tunisia

*Corresponding author: Sahla Sellami, Department of Pediatric Surgery, Hedi Chaker Hospital, university of Sfax, Tunisia, E-mail: $\underline{\text { sahlasellami89@gmail.com }}$

\begin{abstract}
Background: Rhabdoid tumor of the kidney is an uncommon and aggressive tumor characterized by poor outcomes. Given the rarity of this tumor, the diagnosis is still a challenge and the treatment are not well codified. Case report: A 4-month-old girl presented with abdominal mass. Clinical and radiologic explorations suggested nephroblastoma. The diagnosis of rhabdoid tumor was made post operatively based on anatomopathological findings.
\end{abstract}

Keywords: Renal; Rhabdoid; Wilms; Child

Received Date: February 04, 2019; Accepted Date: March 08, 2019; Published Date: March 15, 2019

\section{Introduction}

Rhabdoid tumor of the kidney (RTK) is an aggressive tumor, which occurs in infancy, and early childhood accounts for $2 \%$ of renal tumors in childhood [1]. It was initially described as sarcomatous type of wilms tumor because of the histologic resemblance of its cells to rhabdomyoblast. Rhabdoid tumor is recognized now as a distinct entity. Clinical presentation and radiological explorations do not often differentiate it from a WILMS tumor (WT). Whereas the overall survival rate for WT exceeds $85 \%$, the survival rate for RTK is only $23 \%$ [2]. We present a case of RTK considered initially as nephroblastoma and describe the clinical and radiologic findings and management.

\section{Case Report}

Our patient was preterm born at 34 weeks of gestation via caesarian section to a healthy 34-year-old mother and was conceived through vitro fertilization; the pregnancy was well followed-up and normally unrolled. The prenatal trimestral ultrasound was

Citation: Sahla Sellami, How to Distinguish Non-Metastatic Rhabdoid Tumor from Wilms Tumor in Young Infants Preoperatively: A Case Report and Literature Review. J Clin Cases Rep 2(3): 65-68. DOI: https://doi.org/10.46619/joccr.2019.2-1041

(C) 2019 Tridha Scholars 
normal. No congenital anomalies or malignancies were noticed in the family history. The birth weight was $2.3 \mathrm{~kg}$. The patient was admitted at the age of 4 months in our department for vomiting and fever. The parents said that they noticed an abdominal distension few weeks earlier. The physical examination showed a right lumbar mass. The abdominal ultrasound followed by the CT scan showed a large heterogeneous retroperitoneal mass with scattered calcifications measuring $10 \mathrm{~cm}$. The tumor was predominantly solid and focally cystic with the presence of areas of hemorrhage and necrosis (Figure 1). It was at expense of the right kidney and did not invade vascular structures or nearby organs. Biologic explorations showed anemia, hypercalcemia and normal amounts of catecholamines. The diagnosis of nephroblastoma was highly suspected. The patient didn't undertake pre-operative chemotherapy and had surgical treatment consisting on enlarged nephroureterectomy as recommended by the SIOP. During surgery, the tumor was encapsulated occupying the entire kidney and was intimately adherent to the right renal vein, the inferior vena cava. A total macroscopic resection of the tumor was realized. The patient died after the surgery due to a hemodynamic instability. The macroscopic examination of the specimen showed a large encapsulated tumor at the right kidney and measuring $12 \mathrm{~cm} \times 6 \mathrm{~cm} \times 5 \mathrm{~cm}$. The microscopic examination found sheets of round to oval cells with eosinophilic cytoplasm, large and eccentric nuclei and prominent nucleoli. Numerous mitosis was seen and areas of hemorrhage and necrosis were present. Immunohistochemically, many tumor cells were positive for vimentin and EMA and focally for keratin and cyclin D1. Whereas, immunostaining for AML and Desmin were negative (Figure 1). The diagnosis of RTK was based on these findings.
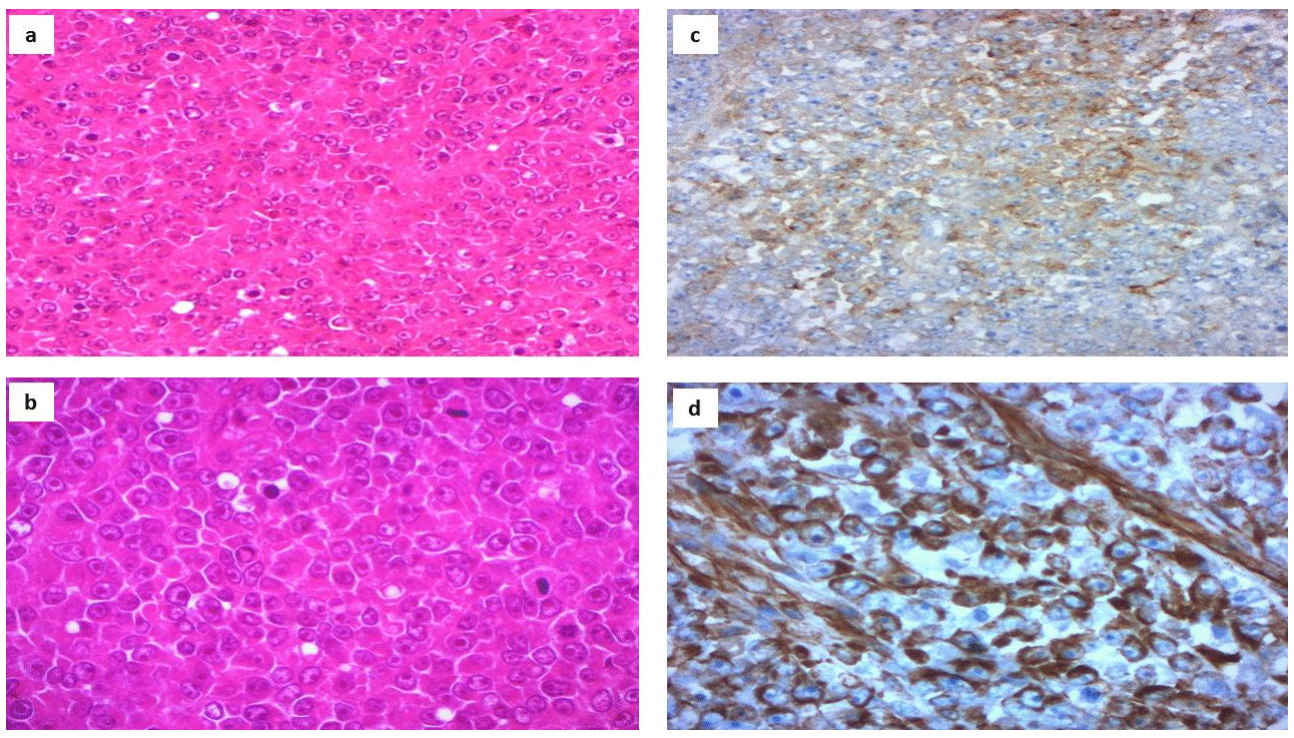

Figure 1: (a) (HEX200), (b) (HE X400): solid nests of round or oval cells with abundant eosinophilic cytoplasm, eccentric nuclei, prominent nucleoli and focally hyaline pink cytoplasmic inclusions. Immunostaining for EMA (c) (X200) and Vimentin (d) were positive (X400).

\section{Discussion}

The RTK is considered one of the most lethal malignancies in pediatric oncology with an overall survival rate of less than $25 \%$. It occurs predominantly during early childhood. Eighty percent of cases are diagnosed at an age less than two years. The median age reported by SIOP was 13 months and by NWTSG was 10,6 months [2]. However, the mean age at diagnosis in wilms tumor is 36 months [2-3]. The primary site of rhabdoid tumor is renal at a rate of $45 \%$, the central nervous system at a rate of $18 \%$ and other tissue at a rate of $35 \%$ [4]. Epidemiology features suggest that in vitro fertilization, preterm birth and 
http://www.tridhascholars.org | July-2019

low birth weight are associated with a higher risk of occurrence of the rhabdoid tumor [5,6]. Our patient had all these features but in front of the age and radiologic findings the diagnosis of wilms was evoked. The discovery of an abdominal mass during physical examination is the most constant clinical sign, but this cannot guide the diagnosis. Some clinical features can suggest the diagnosis of RTK from other tumors: fever, hematuria, a young age and high tumor stage [7]. Rhabdoid tumors can be seen in the newborn and are in this case, possibly revealed by subcutaneous tumor nodules [8]. Symptoms may also be preferable to metastasis. Common metastatic site includes the lung, the brain and bone. The biological assessment can reveal abnormalities such as anemia and hypercalcemia [7,9]. Hypercalcemia is rare in case of wilms tumor however it occurs in approximately $20 \%$ of rhabdoid tumors [3]. It is attributed to the ectopic production of parathyroid hormone related protein by the tumor. Our patient presented persistent hypercalcemia needing a preoperative correction during 2 weeks consisting on hydration and bisphosphonate therapy. This hypercalcemia was considered as a paraneoplastic syndrome. In our case the diagnosis of mesoblastic nephroma was initially evoked accounting for the age of patients. However subsequent radiologic features eliminated this diagnosis. In radiological explorations, rhabdoid tumors appear similar to nephroblastoma but certain features can orient the diagnosis: subcapsular fluid collection with areas of hemorrhage and necrosis is more correlated with a rhabdoid tumor (71\% vs. $12 \%$ of wilms tumors) [10,11]. A multilobulated architecture especially when surrounded by low attenuation material, a sign of hemorrhage and necrosis, is more present in rhabdoid tumor [11-13] and calcifications are encountered in $66 \%$ of rhabdoid tumors vs. 6 to $15 \%$ of wilms tumors $[11,13,14]$. Calcifications and necrosis were present in our case but considering the fact that there were no specific signs and given the rarity of rhabdoid tumors, it was considered as Wilms tumor.

Seventy per cent of patients had advanced stage at the time of diagnosis [2-4] and $24 \%$ had metastasis [3]. In the case of our patient, the tumor would have been classified as stage I.

Pathological study shows in the macroscopic examination that the tumors are typically large, hemorrhagic, and necrotic, with poorly defined borders that reflect their highly invasive nature. Histologically, the tumor is composed of sheets of relatively uniform round to polygonal cells with rhabdoid features. These features consist of vesicular chromatin, prominent nucleoli and hyaline pink cytoplasmic inclusions. Immunohistochemically, it is characterized by a positivity for vimentin and cytokeratin and a negativity for INI1 which is a sensitive and specific marker of these neoplasms [15].

The prognosis is related to the age at the time of diagnosis $[2,3,16]$. Diagnosis at an early stage and the use of radiotherapy are also associated with better outcomes [17]. The preoperative chemotherapy does not seem to improve the survival rate of patients with RTK $[2,3,18]$ even if it seems to decrease the tumor volume [3]. Given the rarity of this tumor there is no standard treatment option for RTK [19-20]

\section{Conclusion}

To conclude epidemiologic features such as low birth weight, age less than 2-year, history of in vitro fertilization, biologic elements such as hypercalcemia, anemia and calcifications in CT Scan may orientate to the diagnosis of rhabdoid tumor, and should include in a diagnostic score that would guide the therapeutic attitude.

\section{References}


http://www.tridhascholars.org | July-2019

1. Argani P (2004) Rhabdoid tumour In: World Health Organization Classification of Tumours Pathology \& Genetics Tumours of the Urinary System and Male Genital Organs. Eble JN, Sauter G, Epstein IJ, et al. (Eds.). IARC Press, Lyon 58- 59.

2. Tomlinson G, Breslow N, Dome J, et al. (2005) Rhabdoid tumor of the kidney in the National Wilms' Tumor Study: Age at diagnosis as a prognostic factor. Journal of Clinical Oncology 23: 7641-7645.

3. van den Heuvel-Eibrink MM, van Tinteren H, Rehorst H, et al. (2011) Malignant rhabdoid tumors of the kidney (MRTKs), registered on recent SIOP protocols from 1993 to 2005: a report of the SIOP renal tumour study group. Pediatric Blood Cancer 56(5): 733-737.

4. Reinhard H, Reinert J, Beier R, et al. (2008) Rhabdoid tumors in children: Prognostic factors in 70 patients diagnosed in Germany. Oncology Reports 19(3): 819-823.

5. Heck JE, Lombardi CA, Cockburn M, et al. (2013) Epidemiology of rhabdoid tumors of early childhood. Pediatric Blood \& Cancer 60: 77-81.

6. Cecen E, Gunes D, Uysal KM, et al. (2010) Atypical teratoid/rhabdoid tumor in an infant conceived by in vitro fertilization. Childs Nervous System 26(2): 263-266.

7. Amar A, Tomlinson G, Green D, et al. (2001) Clinical presentation of rhabdoid tumors of the kidney. Journal of Pediatric Hematology/Oncology 23(2): 105-108.

8. Hsueh C, Kuo TT (1998) Congenital malignant rhabdoid tumor presenting as a cutaneous nodule: report of 2 cases with review of the literature. Archives of Pathology \& Laboratory Medicine 122(12): 1099-1102.

9. Weeks DA, Beckwith JB, Mierau GW, et al. (1989) Rhabdoid tumor of kidney. A report of 111 cases from the national Wilms' tumor study pathology center. The American Journal of Surgical Pathology 13: 439-458.

10. Chung EM, Graeber AR, Conran RM (2016) Renal tumors of childhood: radiologic-pathologic correlation Part I. The $1^{\text {st }}$ decade. Radiographics 36(2): 499-522.

11. Agrons GA, Kingsman KD, Wagner BJ, et al. (1997) Rhabdoid tumor of the kidney in children: a comparative study of 21 cases. AJR. American Journal of Roentgenology 168(2): 447-451.

12. Chung CJ, Lorenzo R, Rayder S, et al. (1995) Rhabdoid tumors of the kidney in children: CT findings. AJR. American Journal of Roentgenology 164(3): 697-700.

13. Han TI, Kim MJ, Yoon HK, et al. (2001) Rhabdoid tumour of the kidney: imaging findings. Pediatric Radiology 31(4): 233-237.

14. Prasad SR, Humphrey PA, Menias CO, et al. (2005) Neoplasms of the renal medulla: radiologic-pathologic correlation. RadioGraphics 25(2): 369-380.

15. Argani P, Dehner L, Leuschner I (2016) Mesenchymal tumors occurring mainly children In: Moch H, Humphrey PA, Ulbright TM, Reuter VE (Eds.). WHO classification of tumors of the urinary system and male genital organs. Lyon: International Agency for Research on Cancer 55-56.

16. Brennan B, Foot A, Stiller C, et al. (2004) Where to next with extracranial rhabdoid tumors in children. European Journal of Cancer 40(4): 624-626.

17. Sultan I, Qaddoumi I, Rodríguez-Galindo C, et al. (2010) Age, stage, and radiotherapy, but not primary tumor site, affects the outcome of patients with malignant rhabdoid tumors. Pediatric Blood \& Cancer 54(1): 35-40. 
http://www.tridhascholars.org | July-2019

18. Furtwängler R, Kager L, Melchior P, et al. (2018) High-dose treatment for malignant rhabdoid tumor of the kidney: No evidence for improved survival-The Gesellschaft für Pädiatrische Onkologie und Hämatologie (GPOH) experience. Pediatric Blood \& Cancer 65(1).

19. Furtwängler R, Kager L, Melchior P, et al. (2018) High-dose treatment for malignant rhabdoid tumor of the kidney: No evidence for improved survival-The Gesellschaft für Pädiatrische Onkologie und Hämatologie (GPOH) experience. Pediatric Blood Cancer 65(1): 2018.

20. Furtwängler R, Nourkami-Tutdibi N, Leuschner I, et al. (2014) Malignant rhabdoid tumor of the kidney: significantly improved response to pre-operative treatment intensified with doxorubicin. Cancer Genetics 207(9): 434-436. 